\title{
Can laparoscopic cystectomy become the method of choice in the treatment of invasive urothelial urinary bladder cancer?
}

\author{
Przemysław Adamczyk ${ }^{1, A, C, D, F}$, Kajetan Juszczak ${ }^{2, C, E}$, Mateusz Kadłubowski ${ }^{1, B}$, Adam 0strowski ${ }^{3, B}$, Piotr Maciukiewicz ${ }^{2, E}$, Tomasz Drewa ${ }^{3, F}$ \\ ${ }^{1}$ Department of General and Oncologic Urology, Nicolaus Copernicus Hospital in Torun, Poland \\ 2 Department of Urology, Memorial Rydygier Hospital, Kraków, Poland \\ ${ }^{3}$ Clinic of General and Oncologic Urology, Collegium Medicum of Nicolaus Copernicus University, Bydgoszzz, Poland \\ A - research concept and design; B - collection and/or assembly of data; $\mathrm{C}$ - data analysis and interpretation; \\ $D$ - writing the article; $E$ - critical revision of the article; $F$ - final approval of the article
}

\section{Address for correspondence}

Przemysław Adamczyk

E-mail: przemekad@poczta.onet.pl

Funding sources

None declared

Conflict of interest

None declared

Received on April 15, 2019

Reviewed on September 8, 2019

Accepted on December 5, 2019

Published online on March 24, 2020

Cite as

Adamczyk P, Juszczak K, Kadłubowski M, Ostrowski A, Maciukiewicz P, Drewa T. Can laparoscopic cystectomy become the method of choice in the treatment of invasive urothelial urinary bladder cancer? Adv Clin Exp Med. 2020;29(3):301-306. doi:10.17219/acem/115084

DOI

10.17219/acem/115084

Copyright

Copyright by Author(s)

This is an article distributed under the terms of the

Creative Commons Attribution 3.0 Unported (CC BY 3.0)

(https://creativecommons.org/licenses/by/3.0/)

\begin{abstract}
Background. Radical cystectomy with pelvic lymphadenectomy is the method of choice for muscle-invasive urothelial cell cancer (UCC) treatment and provides the best cancer-specific survival. It can be performed as an open radical cystectomy (ORC), laparoscopic radical cystectomy (LRC) or robot-assisted surgery (RARC).

Objectives. The aim of this study was to compare laparoscopic and open radical cystectomy in terms of perioperative and oncological results.

Material and methods. This retrospective study included 260 patients who underwent surgery due to invasive bladder cancer. A laparoscopic radical cystectomy (LRC) was performed on 131 patients and an open radical cystectomy (ORC) on 129 patients. Group was stratified according to the urinary diversion. Oncologic results expressed as perioperative variables were analyzed, adjusted to the type of urinary diversion.

Results. The LRC patients were in worse perioperative condition according to the American Society of Anesthesiologists (ASA) score than the ORC group (3.1 and 2.52, respectively; $p=0.001$ ). The serum protein level was significantly lower in the ORC group, with no difference in body mass index (BMI) between the groups. The median operation time was significantly shorter in the LRC group with ileal conduit and uretero-cutaneostomy than in the same groups operated using open approach (252.5 min and $180 \mathrm{~min}$ vs $290 \mathrm{~min}$ and 225 min, respectively), as was the hospital discharge time (8.18 days and 11.63 days, respectively; $p=0.004$ ). In both LRC groups, median blood loss was lower, compared with corresponding ORC groups ( $325 \mathrm{~mL}$ and $400 \mathrm{ml}$ vs $800 \mathrm{~mL}$ and 1,100 $\mathrm{mL}$, respectively; $p<0.001$ in both cases). The level of complications was significantly lower in both $L R C$ groups than in the ORC groups ( $p<0.001$ and $p=0.001$, respectively). The lymph node yield was 12 in the LRC group and 10 in the ORC group. The LRC group had a lower positive surgical margins ratio.
\end{abstract}

Conclusions. The laparoscopic approach should be a valid option for radical cystectomy, given the fewer complications, smaller blood loss, and shorter operating and hospitalization times experienced by patients who underwent a laparoscopic cystectomy.

Key words: laparoscopy, cystectomy, urinary bladder neoplasms, cystectomy methods 


\section{Introduction}

Urothelial cell cancer (UCC) is one of most common neoplasms that invade the genitourinary tract. ${ }^{1}$ There are 380,000 new cases each year worldwide and more than 150,000 deaths per year. ${ }^{2}$ Radical cystectomy (RC) with pelvic lymphadenectomy, together with neoadjuvant or adjuvant chemotherapy for advanced cases, is the method of choice for muscle-invasive UCC and provides the best cancer-specific survival. ${ }^{3}$ After a cystectomy, the 10-year recurrence-free survival rate is $50-59 \%$, and the overall survival rate is around $45 \% .^{2,4}$ Radical cystectomy can be performed as an open surgery (ORC), a laparoscopic surgery (LRC) or a robot-assisted surgery (RARC).

Open radical cystectomy can be associated with a clinically significant number of perioperative complications and a prolonged recovery time. ${ }^{5}$ Since it is a highly morbid procedure, a minimally invasive approach was proposed. ${ }^{6}$ Laparoscopic radical cystectomy was introduced in 1993 by de Badajoz et al. ${ }^{7}$ who performed it for the first time for an oncologic indication. This method reduces blood loss, analgesic consumption and postoperative complications; it also allows the patient to recover bowel function earlier and to return to normal activity. ${ }^{8}$ On the other hand, LRC has a long learning curve and requires a large amount of effort for it to become an established method. More recently, Menon et al. reported the first series of RARC, a procedure that has since gained wide popularity. ${ }^{9}$ A recently published metaanalysis showed an even significantly lower rate of complications 30 days and 90 days after RARC than ORC. When using this minimally invasive surgical approach, the number of complications was significantly lower in groups with grade 4 and 5 complications. The RARC group had a longer operating time but the advantages were lower blood loss and a shorter hospital stay than the ORC group. ${ }^{10}$

The RARC is a demanding procedure and has a long learning curve. It is associated with high costs, which secondary or tertiary urological centers cannot afford. On the other hand, the equipment required for LRC is less expensive and can be afforded by most hospitals worldwide. ${ }^{10}$ The benefits and lower costs associated with LRC suggest that it is a valid alternative that should be developed, despite the existence of a more modern robotic approach.

There are not many head-to-head trials comparing LRC and ORC. Since it is more widely used, ${ }^{11}$ it seems that LRC is slowly becoming an acceptable alternative to ORC. In this study, we explored the safety and efficacy of LRC compared to ORC in terms of perioperative and oncological results.

\section{Material and methods}

In this retrospective study, we analyzed the data from 260 consecutive patients from 2 institutions who received
RC between 2012 and 2016. Of these, 129 received an ORC and 131 received an LRC. The choice of approach (open or laparoscopic) was based on surgeons' choice and their confidence in laparoscopic surgery. The surgical procedure included the removal of the prostate gland in male patients and the reproductive system in female patients. The obturator, external, internal, common iliac, and presacral lymph nodes were dissected for pathological analysis according to the procedures described in Campbell-Walsh Urology. ${ }^{12}$ Indications for cystectomy were, according to the guidelines from the European Association of Urology (EAU), urothelial cT2N0M0-cT4aN0M0 disease or noninvasive papillary cancer that could not be controlled with transurethral resection.

This research project was carried out according to the Declaration of Helsinki.

All patients underwent a preoperative examination, which included routine laboratory tests, a chest radiogram, an abdominal ultrasonography (USG), and a computed tomography (CT) scan or magnetic resonance imaging (MRI). Age, gender, body mass index (BMI), comorbidities, surgical history, and laboratory test results were collected. The operating time (defined as the duration of anesthesia), estimated blood loss and transfusion rates were also collected. Anesthesia risk was assessed and scored according to the American Association of Anesthesiology (ASA) Physical Status Classification System. The nutritional status of patients was assessed using the Nutritional Risk Screening 2002 scale. ${ }^{13}$ Oncologic variables and results were noted, and neoplasm staging was done according to the TNM (tumor-nodule-metastasis) classification system of the Union Internationale Contre le Cancer. ${ }^{14}$ The clinical outcomes were analyzed according to the following definitions: 1) perioperative mortality: any death within 30 days after the surgery, 2) early complications: occurring within 90 days after cystectomy and 3) late complications occurring $>90$ days after cystectomy. ${ }^{15}$ Complications were classified according to the Clavien-Dindo classification system. ${ }^{16}$ Both groups were divided into 3 subgroups according to the urine derivation (ileal conduit, orthotopic neobladder according to Studer, and simple uretero-cutaneostomy). All perioperative data was analyzed according to those variables.

\section{Statistical analysis}

The results were expressed as median, mean and standard deviation $( \pm S D)$. Comparisons between groups of nonparametric data were performed using the MannWhitney U test and the Kruskal-Wallis test as appropriate. Dunn's test was used for post-hoc comparisons, with the Benjamini-Hochberg method for false discovery rate correction. Statistical significance was set at $\mathrm{p} \leq 0.05$ for all tests. 


\section{Results}

\section{Preoperative status evaluation}

There was a significant difference in the risk of anesthesia between the LRC and ORC groups (ASA score 3.1 and 2.52 , respectively; $\mathrm{p}=0.001 ;$ Fig. 1 ). No statistical difference was found in the BMI between the LRC and ORC groups $\left(26.21 \mathrm{~kg} / \mathrm{m}^{2}\right.$ and $25.65 \mathrm{~kg} / \mathrm{m}^{2}$, respectively; $\left.\mathrm{p}=0.11\right)$, but the serum protein level was statistically different $(7.10 \mathrm{~g} / \mathrm{L}$ and $5.56 \mathrm{~g} / \mathrm{L}$, respectively; $\mathrm{p}=0.001$ ).

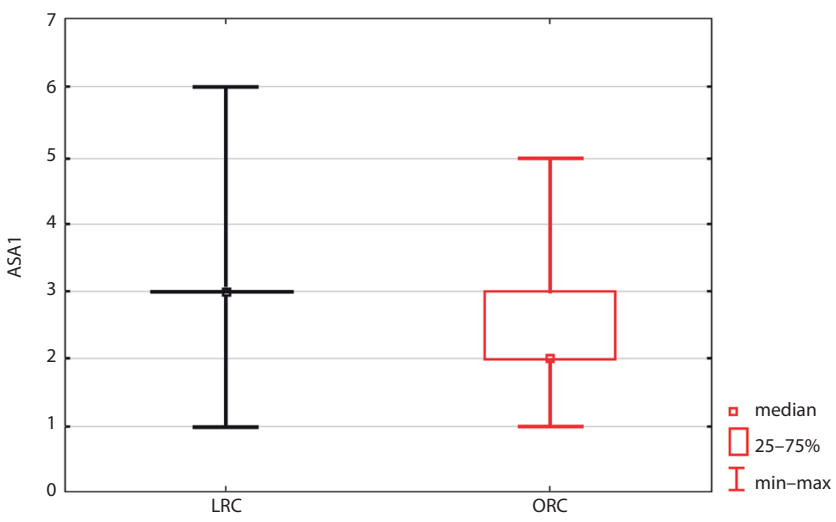

Fig. 1. Mean ASA score in the LRC vs ORC group

\section{Surgical procedure evaluation}

Uretero-cutaneostomy was performed in older and compromised patients, while younger ones received an ileal conduit or Studer neobladder (Table 1).

Table 1. Urine derivation after radical cystectomy

\begin{tabular}{|l|c|c|c|c|}
\multirow{2}{*}{ Group } & \multicolumn{4}{|c|}{ Urine diversion } \\
\cline { 2 - 5 } & $\begin{array}{c}\text { ileal } \\
\text { conduit }\end{array}$ & $\begin{array}{c}\text { orthotropic } \\
\text { neobladder }\end{array}$ & $\begin{array}{c}\text { uretero- } \\
\text { cutaneostomy }\end{array}$ & other \\
\hline LRC & 36 & 0 & 93 & 2 \\
\hline ORC & 71 & 31 & 26 & 1 \\
\hline
\end{tabular}

LRC - laparoscopic radical cystectomy; ORC - open radical cystectomy.
The median operating time was significantly shorter in the LRC group than in the ORC group (190 min vs 290 min; $p=0.001)$. In the case of both groups operated using laparoscopic approach (group with urine derivation using uretero-cutaneostomy and ileal conduit), the median time of surgery was shorter compared to the same groups operated using open approach. It was significantly shorter in the laparoscopic (LAP) group with uretero-cutaneostomy ( $p=0.0016)$ than in the group in which the open technique was used, and almost reached significance in the LAP group with ileal conduit ( $\mathrm{p}=0.067)$ compared to the group where the open technique was used (Table 2).

The median blood loss was significantly lower in the LRC group (350 mL) than in the ORC group $(850 \mathrm{~mL}$; $\mathrm{p}=0.0001)$. In the case of both groups operated using laparoscopic approach (group with urine derivation using uretero-cutaneostomy and ileal conduit), the median blood loss was significantly lower compared to the same groups operated using open approach ( $\mathrm{p}<0.001$ in both cases).

The mean hospital stay for the LRC group was 8.18 days and for the ORC group 11.63 days. Patients from the LRC group required a statistically significant shorter time before being released home $(\mathrm{p}=0.004)$.

\section{Complications}

Most perioperative complications were classified as level 1 and 2 in the LRC group and as level 2 in the ORC group. The mean Clavien-Dindo score was significantly lower in the LRC group than in the ORC group (2.24 vs 2.65; $\mathrm{p}=0.0001)$.

Only 1 patient in the LRC group required conversion to open surgery as a result of neoplasm infiltration of the rectal wall that required resection of the rectum. Both groups included patients that required reoperation. In the ORC group, 8 patients required reoperation: 2 because of urine leakage, infection and peritonitis; 4 due to eventration; 1 due to ileal conduit leakage and re-anastomosis; and 1 due to extensive bleeding. In the LRC group, 9 patients required reoperation: 1 due to eventration; 1 due to incidental loss of urethral drainage; 3 due to ileus and

Table 2. Surgical results adjusted to urine derivation after radical cystectomy

\begin{tabular}{|c|c|c|c|c|c|c|}
\hline Variable & $\begin{array}{l}\text { LRC+ } \\
\text { ileal conduit }\end{array}$ & $\begin{array}{l}\text { LRC+ uretero- } \\
\text { cutaneostomy }\end{array}$ & $\begin{array}{l}\text { ORC+ } \\
\text { ileal conduit }\end{array}$ & $\begin{array}{c}\text { ORC+ } \\
\text { orthotopic } \\
\text { neobladder }\end{array}$ & $\begin{array}{l}\text { ORC+ uretero- } \\
\text { cutaneostomy }\end{array}$ & p-value* \\
\hline Number of patients & 36 & 93 & 71 & 31 & 26 & \\
\hline $\begin{array}{l}\text { Operation time [min] } \\
\text { (median }(\mid \mathrm{QQR}) \text { ) }\end{array}$ & $\begin{array}{c}252.50 \\
(237.50,290.00)\end{array}$ & $\begin{array}{c}180.00 \\
(155.00,200.00)\end{array}$ & $\begin{array}{c}290.00 \\
(260.00,315.00)\end{array}$ & $\begin{array}{c}325.00 \\
(300.00,360.00)\end{array}$ & $\begin{array}{c}225.00 \\
(180.00,275.00)\end{array}$ & $<0.001$ \\
\hline $\begin{array}{l}\text { Blood loss [mL] } \\
\text { (median (IQR)) }\end{array}$ & $\begin{array}{c}325.00 \\
(200.00,400.00)\end{array}$ & $\begin{array}{c}400.00 \\
(250.00,600.00)\end{array}$ & $\begin{array}{c}700.00 \\
(500.00,1000.00)\end{array}$ & $\begin{array}{c}800.00 \\
(600.00,900.00)\end{array}$ & $\begin{array}{c}1,100.00 \\
(500.00,1,500.00)\end{array}$ & $<0.001$ \\
\hline $\begin{array}{l}\text { Complication rate } \\
\text { (median (IQR)) }\end{array}$ & $\begin{array}{c}1.00 \\
(1.00,2.00)\end{array}$ & $\begin{array}{c}2.00 \\
(1.00,2.00)\end{array}$ & $\begin{array}{c}2.00 \\
(2.00,2.00)\end{array}$ & $\begin{array}{c}2.00 \\
(2.00,2.00)\end{array}$ & $\begin{array}{c}2.00 \\
(2.00,2.50)\end{array}$ & $<0.001$ \\
\hline
\end{tabular}

* Kruskal-Wallis test; IQR - interquartile range; LRC - laparoscopic radical cystectomy; ORC - open radical cystectomy. Complication rate according to Clavien-Dindo scale. 
urine leakage followed by infection; and 3 due to urine leakage and ileal conduit re-anastomosis.

Patient deaths in the perioperative period occurred in both groups: 5 in the LRC group and 4 in the ORC group. Four of them were due to intestinal complications (ileus) that led to more serious complications and reoperations. One was due to acute cholecystolithiasis, which required an operation and then had surgical complications. Another death was a result of urine leakage and an infection, followed by sepsis and septic shock. One other case was a result of excessive bleeding with serious cardiovascular complications. Finally, 2 deaths were due to cardiovascular complications.

In the case of both groups operated using laparoscopic approach (group with urine derivation using ureterocutaneostomy and ileal conduit), the median ClavienDindo complication rate was significantly lower, compared to the same groups operated using open approach $(\mathrm{p}=0.001$ and $(\mathrm{p}<0.001$, respectively).

\section{Oncologic results}

Pathologic evaluation revealed urothelial carcinoma in different stages of the disease. In the LRC group, 60\% of patients were diagnosed with pT3-4 disease (Fig. 2). In the ORC group, the percentage of patients with highand very high-risk disease was similar (59\%; Fig. 3).

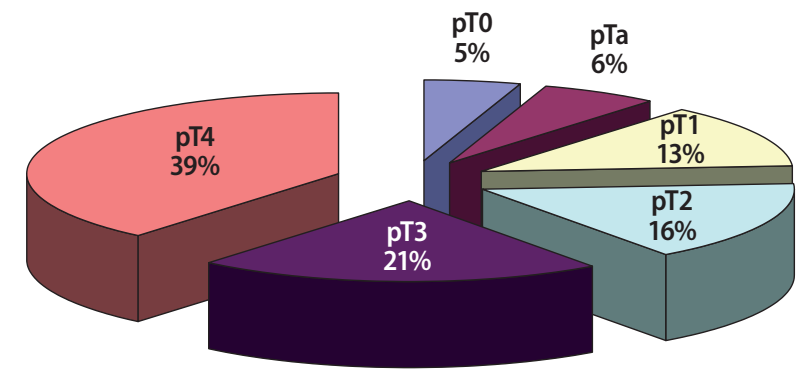

Fig. 2. Pathological stage of disease in patients operated using LRC

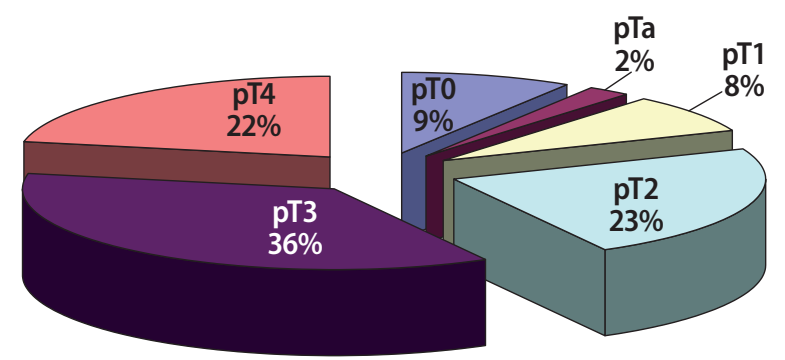

Fig. 3. Pathological stage of disease in patients operated using ORC
Nodal involvement was observed in $26 \%$ of patients from the LRC group and 36\% from the ORC group, and the mean number of nodes taken per patient was 10 and 12 , respectively. There was no statistical difference between the groups.

Positive surgical margins (PSM) were observed in both groups, without significant differences in most instances. The exception was ureteral PSM, with 10 instances in the ORC group and 4 in the LRC group ( $\mathrm{p}=0.04$; Table 3 ).

\section{Discussion}

The classical treatment for urothelial muscle-invasive bladder cancer (MIBC) is ORC. According to the 2016 EAU guidelines, this approach should be proposed to all patients, since the cure rate is highest compared with other methods used alone (radiotherapy, chemotherapy). ${ }^{2}$ Open radical cystectomy provides the longest cancer-specific survival and is considered the standard method of treatment. Since the end of the $20^{\text {th }}$ century, LRC has gained wide popularity. It is less invasive and causes less blood loss and a lower number of perioperative complications. It provides good cancer control and similar oncological outcomes to ORC. ${ }^{8,11}$ There are very few comparative prospective trials comparing ORC and LRC. Up to now, mostly comparative retrospective analyses have been conducted, which have showed no inferiority of LRC vs ORC. A report from a comparative study indicates a recurrence-free survival of $77 \%$ after LRC compared with $80 \%$ after ORC in a period of 3-4 years, but the difference was not statistically significant $(\mathrm{p}=0.2) .{ }^{17}$ Unfortunately, the analyzed series was small, consisting of only 55 patients.

In our study, we included 260 patients, which seems to be the highest number of patients analyzed so far. Only Huang et al. included a comparable number of patients in their report (108). ${ }^{18}$

Local recurrence, PSM, lymph node yield, and recurrence-free survival are the most important oncologic variables. In available studies, the local recurrences, lymph node yield and cancer-free survival were similar in LRC and ORC patients. This indicates that, from an oncologic point of view, both methods are comparable. ${ }^{19}$ An interesting meta-analysis of 16 papers showed no difference in lymph node yield between LRC and ORC groups. Furthermore, there were fewer positive lymph node invasions and a significantly lower PSM rate in LRC patients $\left(\mathrm{p}=0.05\right.$ and $\mathrm{p}=0.006$, respectively). ${ }^{20}$ In our study, PSM

Table 3. Positive surgical margins in the ORC group vs the LRC group

\begin{tabular}{|l|c|c|c|c|}
\multicolumn{1}{c|}{ Group } & Ureteral PSM & Urethral PSM & $\begin{array}{c}\text { Uterus or prostate } \\
\text { involvement }\end{array}$ & Perivesical fat \\
\hline LRC & 4 & 14 & 11 & 52 \\
\hline ORC & 10 & 10 & 24 & 57 \\
\hline
\end{tabular}

LRC - laparoscopic radical cystectomy; ORC - open radical cystectomy; PSM - positive surgical margins. 
were found in both groups, but the difference was statistically significant only in the case of ureteral PSM.

Oncological safety, defined by the presence of PSM and the number of lymph nodes removed, was questioned in a few older series. ${ }^{21}$ Herr et al. proposed that if $10-14$ lymph nodes were removed and the PSM were less than $10 \%$, the oncologic results of LRC could be considered equivalent to those of ORC. ${ }^{22}$ In our study, the mean number of lymph nodes removed was 10 in the LRC group and 12 in the ORC group, which was sufficient for oncologic staging. Surgical margins were positive in 18 of $129(13.95 \%)$ patients in the ORC group and 15 of 131 (11.4\%) patients in the LRC group. This is slightly higher than the accepted cumulative PSM ratio, but it is generally positive in highvolume disease (pT4). ${ }^{22}$ Among the patients with lower-risk disease (pT2 and lower), none had PSM. The higher percentage of PSM in the ORC group than in the LRC group can suggest, that the PSM percentage does not depend on the surgical approach but more on the local advancement of the disease. Therefore, both methods can be considered similar regarding oncologic safety.

Laparoscopic radical cystectomy is a demanding procedure, but our data suggests that it can also be used to treat localized MIBC. In our study, the learning curve was not that long. After 50 cases, it was possible to achieve operating times, intraoperative blood loss, and the length of hospital stays comparable, or better, than those reported in the largest published ORC series. ${ }^{23}$ It seems that in experienced hands, the operating time can be shorter than for ORC. Such short operating times can be achieved only by an experienced laparoscopic team. At the beginning of the LRC program, our team was well-experienced in laparoscopic prostatectomy and nephrectomy, so the learning curve was much shorter than expected. During LRC, there is a better visualization of all the anatomical structures (particularly in the pelvis minor) than in the classical open surgery. In experienced hands, LRC is easier and more manageable than ORC. It seems that with improved skills and experience on the part of surgeons, LRC could replace ORC.

With the emergence of robotics surgery, RARC is rapidly replacing laparoscopy. ${ }^{24}$ The learning curve is shorter and postoperative results are comparable. In a comparative analysis between LRC and RARC, Abraham et al. concluded that both can be performed safely with good oncological results. ${ }^{24}$ However, RARC is more expensive and cannot be used in secondary or tertiary urological departments. Blood loss was significantly lower in the LRC group. Only 18 out of 131 patients needed transfusions, and only 34 blood units were required in total. In comparison, $80 \mathrm{pa-}$ tients out of 129 needed transfusions in the ORC group, and 198 blood units were used. The same significant results were noted when recorded in subgroups, adjusted for type of urine derivation. This could be expected since the highest blood loss is noted usually during bladder resection, which is a part of radical cystectomy, rather than during urine derivation. The same trend was observed in a large meta-analysis by Tang et al., where the blood loss and transfusion rate from 16 studies were significantly lower in the LRC group than in the ORC group ( $\mathrm{p}=0.001$ and $\mathrm{p}=0.002$, respectively). ${ }^{20}$ It seems that better visualization and greater comfort during surgery improves hemostasis. Hospital stay is one of the most noticeable differences for patients. Recent, unpublished data from our team shows that the advantages of LRC together with the Early Recovery After Surgery (ERAS) program, enable safe hospital discharge 5-7 days after surgery. In this study with older data, the hospital stay was 8.18 days in the LRC group and 11.63 days in the ORC group. The simpler urine derivation generally used in the LRC group could partially explain such a difference. However, when the length of the hospital stay was calculated for the LRC subgroup of patients with ileal conduit, the difference was almost the same. A shorter hospital stay has also been noticed in large series. ${ }^{25,26}$ It significantly reduced the cost of hospitalization but was possible only with the aid of outpatient department control.

A lower need for analgesia was also observed in the LRC group. In the previously mentioned meta-analysis, the need for analgesia in the LRC group was also significantly lower compared to the ORC group ( $\mathrm{p}=0.001)$. With the implementation of the ERAS program, most patients can get out of bed on the $2^{\text {nd }}$ or $3^{\text {rd }}$ day after surgery and are encouraged to do so.

The rate of perioperative complications is one of the most important markers of surgical safety. In this study, complications were predominantly level 2 in the ORC group and level 1 and 2 in the LRC group. Conversion from LRC to ORC was not common. It happened only in one case, where the disease load was very high and made LRC too risky. The same significant results were noted when recorded in 2 subgroups adjusted for type of urine derivation. It is interesting that even when LAP subgroup with ileal conduit was compared to the subgroup with open simple ureterocutaneostomy, the number of complications showed the superiority of LAP technique $(p=0.0004)$, even when complications usually appeared with more complicated urine derivation. Most life-threatening complications are associated with urine leaks and with later intestinal complications due to urine contact with bowel anastomosis. ${ }^{27}$ For this reason, uretero-cutaneostomy was considered in the cases of very high disease load or poor general status. In the LRC group, the general condition of patients was more serious than in the ORC group, and the mean ASA score was also significantly higher $(\mathrm{p}=0.001)$. Older patients with ASA scores of III and IV were subjected to minimally invasive surgery with great success, with the same level of perioperative safety as in the ORC group. From our unpublished data, it seems that even older patients (above 80 years) with serious comorbidities can be operated safely using the laparoscopic approach.

Laparoscopic radical cystectomy is safe to treat advanced urothelial disease, with similar oncological results and 
faster recovery compared to ORC. After 50 cases in experienced hands, LRC was at least as easy to perform as ORC. The level of perioperative complications, lower blood loss and faster hospital discharge time seen in the LRC group, lead to the conclusion that this technique can be developed in secondary and tertiary urological centers as the method of choice for patients with advanced urothelial disease.

In general, during the learning curve of LRC, this approach seems very difficult to manage and is applicable only in selected cases. However, after 50 operations, LRC was easier to perform than ORC, and difficult cases could be managed just as easily.

\section{ORCID iDs}

Przemysław Adamczyk (10) https://orcid.org/0000-0002-7217-841X Kajetan Juszczak (1) https://orcid.org/0000-0003-0354-0822 Mateusz Kadłubowski (10 https://orcid.org/0000-0001-9376-3231

Adam Ostrowski (1) https://orcid.org/0000-0002-3286-3674

Piotr Maciukiewicz (1) https://orcid.org/0000-0002-2151-3708

Tomasz Drewa (1) https://orcid.org/0000-0001-5347-4136

\section{References}

1. Ferlay J, Steliarova-Foucher E, Lortet-Tieulent J, et al. Cancer incidence and mortality patterns in Europe: Estimates for 40 countries in 2012. Eur J Cancer. 2013;49(6):1374-1403.

2. Babjuk M, Burger M, Zigeuner R, et al; European Association of Urology. EAU guidelines on non-muscle invasive urothelial carcinoma of the bladder: Update 2013. Eur Urol. 2017;71(3):447-461.

3. Grossman HB, Natale RB, Tangen CM, et al. Neoadjuvant chemotherapy plus cystectomy compared with cystectomy alone for locally advanced bladder cancer. N Engl J Med. 2003;349(9):859-866.

4. Cookson MS, Chang SS, Wells N, Parekh DJ, Smith JA Jr. Complications of radical cystectomy for nonmuscle invasive disease: Comparison with muscle invasive disease. J Urol. 2003;169(1):101-104.

5. Boström PJ, Kössi J, Laato M, Nurmi M. Risk factors for mortality and morbidity related to radical cystectomy. BJU Int. 2009;103(2):191-196.

6. Lowrance WT, Rumohr JA, Chang SS, Clark PE, Smith JA Jr, Cookson MS. Contemporary open radical cystectomy: Analysis of perioperative outcomes. J Urol. 2008;179(4):1313-1318.

7. de Badajoz S, Perales G, Rosado RA, de la Cruz GJM, Garrido JA. Radical cystectomy and laparoscopic ileal conduit [in Spanish]. Arch Esp Urol. 1993;46(7):621-624.

8. Guillotreau J, Gamé X, Mouzin M, et al. Radical cystectomy for bladder cancer: Morbidity of laparoscopic versus open surgery. $J$ Urol. 2009;2(181):554-559.

9. Menon M, Hemal AK, Tewari A, Shrivastava A, Shoma AM. Nerve-sparing robot-assisted radical cystoprostatectomy and urinary diversion. BJU Int. 2003;92(3):232-236.
10. Xia L, Wang X, Xu T, et al. Robotic versus open radical cystectomy: An updated systematic review and meta-analysis. PLoS One. 2015; 10(3):e0121032.

11. Albisinni S, Rassweiler J, Abbou CC, et al. Long-term analysis of oncological outcomes after laparoscopic radical cystectomy in Europe: Results from a multicentre study by the European Association of Urology (EAU) section of Uro-technology. BJU Int. 2015;115(6):937-945.

12. Wein A. Campbell-Walsh Urology. $10^{\text {th }}$ ed. Philadelphia, PA: Elsevier Saunders; 2012:2379-2408.

13. Kondrup J, Rasmussen HH, Hamberg O, Stanga Z; Ad Hoc ESPEN Working Group. Nutritional risk screening (NRS 2002): A new method based on an analysis of controlled clinical trials. Clin Nutr. 2003;22(3): 321-336.

14. Sobin LH, Wittekind C. TNM Classification of Malignant Tumours. $6^{\text {th }}$ ed. New York, NY: Wiley-Blackwell; 2002:23-25.

15. Froehner M, Brausi MA, Herr HW, Muto G, Studer UE. Complications following radical cystectomy for bladder cancer in the elderly. Eur Urol. 2009;56(3):443-454.

16. Clavien PA, Barkun J, de Oliviera ML, et al. The Clavien-Dindo classification of surgical complications: Five-year experience. Ann Surg. 2009;250(2):187-196.

17. Hemal AK, Kolla SB. Comparison of laparoscopic and open radical cystoprostatectomy for localized bladder cancer with 3-year oncological follow-up: A single surgeon experience. JUrol. 2007;178(6):2340-2343.

18. Huang J, Huang H, Lin TX, et al. Compare of laparoscopic and open surgery for radical cystectomy with orthotopic ileal neobladder [in Chinese]. Zhonghua Wai Ke Za Zhi. 2008;46(24):1870-1874.

19. Porpiglia F, Renard J, Billia M, et al. Open versus laparoscopy-assisted radical cystectomy: Results of a prospective study. J Endourol. 2007;21(3):325-329.

20. Tang K, Li H, Xia D, et al. Laparoscopic versus open radical cystectomy in bladder cancer: A systematic review and meta-analysis of comparative studies. PLoS One. 2014;16:9(5):e95667.

21. Hautmann RE. The oncologic results of laparoscopic radical cystectomy are not (yet) equivalent to open cystectomy. Curr Opin Urol. 2009;19(5):522-526.

22. Herr H, Lee C, Chang S, Lerner S; Bladder Cancer Collaborative Group. Standardization of radical cystectomy and pelvic lymph node dissection for bladder cancer: A collaborative group report. JUrol. 2004; 171(5):1823-1828.

23. Abboudi $H$, Khan MS, Guru KA, et al. Learning curves for urological procedures: A systematic review. BJU Int. 2014;114(4):617-629.

24. Abraham JB, Young JL, Box GN, Lee HJ, Deane LA, Ornstein DK. Comparative analysis of laparoscopic and robot-assisted radical cystectomy with ileal conduit urinary diversion. J Endourol. 2007;21(12): 1473-1480.

25. Ha US, Kim SI, Kim SJ, Cho HJ, Hong SH. Laparoscopic versus open radical cystectomy for the management of bladder cancer: Mid-term oncological outcome. Int J Urol. 2010;17(1):55-61.

26. Haber G-P, Crouzet S, Gill IS. Laparoscopic and robotic assisted radical cystectomy for bladder cancer: A critical analysis. Eur Urol. 2008;54(1): 54-64.

27. Lawrentschuk N, Colombo R, Hakenberg OW, et al. Prevention and management of complications following radical cystectomy for bladder cancer. Eur Urol. 2010;57(6):983-1001. 